\title{
CHOKING HORNS IN LIPSCHITZ GEOMETRY OF COMPLEX ALGEBRAIC VARIETIES. APPENDIX BY WALTER D. NEUMANN
}

\author{
LEV BIRBRAIR, ALEXANDRE FERNANDES, VINCENT GRANDJEAN, AND DONAL O'SHEA
}

\begin{abstract}
The paper studies the Lipschitz geometry of germs of complex algebraic varieties and introduces the notion of a choking horn. A choking horn is a family of cycles on an algebraic variety with the property that the cycles cannot be boundaries of nearby chains. The presence of choking horns is an obstruction to metric conicalness and the authors use this to prove that some classical isolated hypersurface singularities are not metrically conical. They also show that there exist countably infinitely many singular varieties, which are locally homeomorphic, but not locally subanalytically biLipschitz equivalent with respect to the inner metric. An appendix by W. Neumann uses separating sets to provide another example of the same phenomenon.
\end{abstract}

\section{INTRODUCTION}

Complex analytic subsets of $\mathbb{C}^{n}$ come equipped with two metrics: the outer metric where the distance between two points of the subset is their distance measured in $\mathbb{C}^{n}$ and the inner metric where the distance between two points is the infimum of the length of rectifiable curves in the subset connecting the two points. It has been known for a long time that at a singular point the germ of a complex analytic curve equipped with the inner metric is bi-Lipschitz homeomorphic to finitely many cones over a circle. In other words, the local inner geometry of a complex curve at a singular point is that of a metric cone. This is the geometric analog for curves of the classical result that a complex analytic subset is topologically conical; that is, in the neighborhood of a singular point, it is homeomorphic to the cone over its link.

In higher dimensions, however, it has become apparent that despite being topologically conical at singularities, complex analytic subsets need not be metrically conical. Indeed, a series of recent papers $[3,4,5,6,8]$ have shown that the inner geometry of complex

2010 Mathematics Subject Classification. 14B05, 14J17, 51F99.

Key words and phrases. Lipschitz Geometry, conic and non-conic singularities.

${ }^{a}$ Research supported by CNPq grant no 300575/2010-6.

${ }^{b}$ Research supported by CNPq grant no 302998/2011-0.

${ }^{c}$ Research supported by CNPq grant no 150555/2011-3.

The fourth author was supported by a faculty fellowship and income from the Elizabeth T. Kennan chair, both from Mount Holyoke College, together with support from the mathematics department at UFC in Fortaleza.

The authors wish to thank Alberto Verjovsky and Anne Pichon for valuable conversations and comments. They also thank the referee for a close reading and many helpful comments. 
analytic subsets is both subtle and rich, and have begun to work through the structure and details, mostly in the case of isolated singularities of complex surfaces, of the inner geometry of singularities that are not bi-Lipschitz homeomorphic to some conical subset of Euclidean space. In particular the existence of a fast loop (a 1-cycle bounding a 2chain that contracts the cycle faster than linearly $[3,10,8]$ ) or a separating set (a real hypersurface germ with small density at the singularity which disconnects the germ of the singularity into two or more connected components each of large density at the singularity $[3,6])$ are obstructions to the metric conicalness of the local inner geometry at the singular point.

This paper addresses the following two questions about the local inner geometry of complex analytic singularities:

- First, is it possible to identify objects that obstruct the metric conicalness of the local inner geometry of complex analytic sets near a singular point?

- The Briançon-Speder family [6] is topologically constant but carries two Lipschitz structures. This motivates our second question: how many local inner geometries near the singularities can a fixed homeomorphic type of a complex analytic isolated singularity carry? In particular, can a single topological class have infinitely many Lipschitz models?

In order to answer these questions we introduce a new bi-Lipschitz invariant object, which we call a choking horn. This object is a horn, that is a continuous image of a cylinder over a Euclidean sphere along the unit interval with the property that one boundary sphere maps onto the singular point and the tangent cone of the image at the singular point is a real half-line, with the additional property that the intersection of this image with any Euclidean $(2 n-1)$-sphere of sufficiently small radius centered at the singular point is either a non-trivial cycle in the homology of the link or a trivial cycle which can only be a boundary of a chain with "large" diameter. It turns out that metrically conic singularities cannot admit a choking horn (Theorem 2.6). The choking horns are higher dimensional analogs of so-called fast loops of the second kind, described in the paper of Birbrair, Neumann and Pichon [8]. Moreover, the idea of choking horn is closely related to Metric Homology [1, 2] and Vanishing Homology [15], which study the families of homology cycles (trivial or nontrivial) with respect to the metric properties near the singular points. Moreover this new object can be realized in some families of Brieskorn hypersurface singularities as the real locus of the complex singularity. We show that the presence of choking horns in the family of Brieskorn singularities $\left(A_{2 k+1}^{n}\right)_{k \geq 1}$ also implies that any two disjoint elements of this family cannot have the same local inner geometry at the singular point (Theorem 3.2), despite this family being topologically constant (Theorem 3.3).

Notes: Unless explicitly mentioned otherwise, any subset we will work with always comes equipped with the inner metric. 


\section{Elementary PROPERTies OF CHOKING HORNS}

Given the germ at the origin of a subset $Z$ of an Euclidean space, the $t$-link $Z_{t}$ of $Z$ is the locus of points of $Z$ whose (Euclidean) distance to the origin is equal to $t$.

Let $(X, \mathbf{0})$ be the germ of a connected subanalytic subset of an Euclidean space equipped with a metric $d$ which is either the outer metric $d_{\text {outer }}$, or the inner metric $d_{\text {inner }}$.

Definition 2.1. A horn in $X$ is the image of a subanalytic continuous map

$$
\phi:[0,1] \times \mathbf{S}^{p} \rightarrow X
$$

where $\mathbf{S}^{p}$ is the real unit sphere of positive dimension $p$, and such that there exists a constant $C>0$ such that

1. For any $(t, u) \in[0,1] \times \mathbf{S}^{p}$ the image $\phi(t, u)$ satisfies

$$
\frac{t}{C} \leq d_{\text {outer }}((\phi(t, u)), \mathbf{0}) \leq C t
$$

2. The tangent cone of the image of $\phi$ at $\mathbf{0}$ is just a single real half-line.

We observe that a horn is subanalytic and in particular the mapping $\phi$ collapses the boundary sphere $0 \times \mathbf{S}^{p}$ onto the singular point $\mathbf{0}$.

Definition 2.2. Let $Y$ be horn in $(X, d)$. The subset $Y$ is said to be a choking horn if for every $t$ small enough and any family of chains $\xi_{t}$ (we do not suppose any sort of regularity of the family) such that $\operatorname{supp}\left(\xi_{t}\right) \subset X_{t}$ and $\partial \xi_{t}=Y_{t}$, then the d-diameter of $\operatorname{supp}\left(\xi_{t}\right)$, necessarily, satisfies

$$
\operatorname{diam}\left(\operatorname{supp}\left(\xi_{t}\right)\right) \geq K t
$$

for some real number $K>0$ independent of $t$.

The definition of a choking horn in $X$ depends a priori on the choice of the metric $d$ (outer or inner metric) on $X$. Nevertheless this possible ambiguity is sorted out by the following:

Proposition 2.3. Let $Y$ be a horn in $X$. Then, $Y$ is a choking horn with respect to the inner metric if, and only if, it is a choking horn with respect to the outer metric on $X$.

Proof. Whenever $Y$ is a choking horn with respect to the outer metric then it is a choking horn with respect to the inner metric on $X$. The converse is a direct consequence of Lemma 2.4 below.

Lemma 2.4. There exists a constant $L>0$ such that, for any closed and connected subanalytic subset $Z$ of $X$, the inner-diameter of $Z$ (diameter with respect to the inner metric on $X$ ) is at most $L$ times the outer-diameter of $Z$ (diameter with respect to the outer metric on $X)$. 
Proof. Let $\left\{\Lambda_{i}\right\}_{i=1}^{l}$ be a subanalytic decomposition of the subset $X$ into closed pancakes $[11,12,13,14]$. The existence of such a decomposition ensures there are positive real numbers $\left\{\lambda_{i}\right\}_{i=1}^{l}$ such that, for each $i=1, \ldots, l$, the inner-distance on $\Lambda_{i}$ is at most $\lambda_{i}$ times the outer-distance on $\Lambda_{i}$.

We define the constant $L$ of the statement as $L:=\sum_{i=1}^{l} \lambda_{i}$.

Let $Z$ be a closed connected subanalytic subset of $X$. In order to show that the innerdiameter of $Z$ is at most $L$ times the outer-diameter of $Z$, let $p, q$ be points of $Z$ such that inner-diameter of $Z$ is realized as $d_{\text {inner }}(p, q)$ the inner distance between $p$ and $q$. Since $Z$ is connected, there is a subanalytic arc $\Gamma$ in $Z$ connecting $p$ and $q$. Let $\gamma:[0,1] \rightarrow \Gamma$ be a continuous subanalytic and injective parameterization of the arc $\Gamma$. We can find $k+1 \leq l+1$ points of $\Gamma$

$$
\gamma_{0}:=p, \gamma_{1}:=\gamma\left(t_{1}\right), \ldots, \gamma_{k-1}:=\gamma\left(t_{k-1}\right), \gamma_{k}:=q
$$

such that $0<t_{1}<\ldots<t_{k-1}<1$, and each consecutive pair of points $\gamma_{j-1}$ and $\gamma_{j}$ belong to a same pancake but neither $\gamma_{j-2}$, nor $\gamma_{j+1}$ belongs to this pancake. In other words, $\gamma_{j-1}, \gamma_{j} \in Z \cap \Lambda_{i_{j}}$ for some $i_{j} \in\{1, \ldots, l\}$, but $\gamma_{j-2}, \gamma_{j+1} \notin \Lambda_{i_{j}}$.

Since the inner-distance on $\Lambda_{i}$ is at most $\lambda_{i}$ times the outer-distance on $\Lambda_{i}$, we deduce the following inequalities

$$
\begin{aligned}
d_{\text {inner }}(p, q) & \leq \sum_{j=1}^{k} d_{\text {inner }}\left(\gamma_{j-1}, \gamma_{j}\right) \\
& \leq \sum_{j=1}^{k} \lambda_{i_{j}} d_{\text {outer }}\left(\gamma_{j-1}, \gamma_{j}\right) \\
& \leq \sum_{j=1}^{k} \lambda_{i_{j}} \operatorname{diam}_{\text {outer }}(Z) \\
& \leq L \text { diam }{ }_{\text {outer }}(Z)
\end{aligned}
$$

where $\operatorname{diam}_{\text {outer }}(Z)$ denotes the outer-diameter of $Z$.

We remark that Lemma 2.4 bounding the inner metric diameter from above in terms of the outer metric diameter is what makes the choice of diameter in measuring the size of bounding cycles in the definition of a choking horn attractive. Other measures of size, such as volumes, do not seem to admit such easily accessible bounds. The proposition and lemma above immediately yield the following.

Proposition 2.5. Let $(X, 0)$ be the germ of a connected subanalytic subset of an Euclidean space equipped with the inner metric.

1. The existence of a choking horn in $X$ is invariant by subanalytic bi-Lipschitz homeomorphisms;

2. If $X$ admits a choking horn $Y$ contained in a subgerm $W$, then the subset $Y$ is also a choking horn in $W$. 
We recall that a subanalytic germ at the origin of an Euclidean space equipped with the intrinsic metric is metrically conical if it is bi-Lipschitz homeomorphic to the cone over its link at the origin (where the cone over a subset $\mathrm{K}$ of Euclidean space is the set of points $\{t x: x \in K, t \in[0,1]\})$.

The first result of this note establishes that choking horns are obstructions to the metric conicalness of the local inner geometry at the singularity:

Theorem 2.6. A closed subanalytic metrically conical set-germ $(X, 0)$ does not admit a choking horn.

Proof. By hypothesis we can assume that $X$ is the cone over its link with vertex at $\mathbf{0}$. Let us consider a subanalytic continuous mapping

$$
\phi:[0,1] \times \mathbf{S}^{d} \stackrel{\sim}{\longrightarrow} Y \subset X
$$

parameterizing a horn $Y$ in $X$. Let $Y_{t}$ be the $t$-link of $Y$. Since $X$ is a cone the Hausdorff limit of $\frac{1}{t} Y_{t}$ as $t \rightarrow 0$, taken in the Euclidean unit sphere of the ambient Euclidean space, is a single point corresponding to a point $x$ in the link of $X$. Let us denote the link of $X$ by $L$. Let $B_{\delta}(x)$ denote the Euclidean ball centered at $x$ with radius $\delta>0$. By the topological local structure of subanalytic sets, we know that $B_{\delta}(x) \cap L$ is contractible for all $\delta>0$ small enough. Since $\frac{1}{t} Y_{t}$ is collapsing onto $x$, as $t \rightarrow 0$, we have that Hausdorff distance between $\frac{1}{t} Y_{t}$ and $x$, which we denote by $\delta(t)$, tends to 0 as $t>0$. Moreover, since $\frac{1}{t} Y_{t}$ is a subset of the contractible set $L \cap B_{2 \delta(t)}(x)$, there is a chain $\xi_{t}$ with $\operatorname{supp}\left(\xi_{t}\right) \subset L \cap B_{2 \delta(t)}(x)$ and satisfying $\partial \xi_{t}=\frac{1}{t} Y_{t}$. Since $\operatorname{supp}\left(\xi_{t}\right) \subset L \cap B_{2 \delta(t)}(x)$, we know that $\operatorname{diam}\left(\operatorname{supp}\left(\xi_{t}\right)\right) \rightarrow 0$ as $t \rightarrow 0$.

Now, let us consider the chain $\eta_{t}=t \xi_{t}$ which is in the $t$-link of $X$. Note that,

$$
\partial \eta_{t}=Y_{t} \text { and } \operatorname{diam}\left(\operatorname{supp}\left(\eta_{t}\right)\right)=t \cdot \operatorname{diam}\left(\operatorname{supp}\left(\xi_{t}\right)\right)
$$

hence there is no a positive number $K$ (independent of $t$ ) such that $\operatorname{diam}\left(\operatorname{supp}\left(\eta_{t}\right)\right) \geq K t$ for all $t>0$ small enough. This means exactly that $Y$ does not choke $X$.

\section{3. $X_{k}^{n}$ Brieskorn hypersurface SINGUlarities}

Let $X_{k}^{n}$ be the complex isolated hypersurface singularity of $\mathbb{C}^{n+1}$ defined as

$$
X_{k}^{n}=\left\{\left(z_{1}, \ldots, z_{n}, w\right) \in \mathbb{C}^{n+1}: z_{1}^{2}+\ldots z_{n}^{2}-w^{k}=0\right\}
$$

We are mostly interested in $X_{k}^{n}$ as a germ at the origin $\mathbf{0} \in \mathbb{C}^{n+1}$.

The next theorem shows that choking horns exist in the simplest Brieskorn singularities.

Theorem 3.1. For each $n>1$ and each $k>2$, the hypersurface $X_{n}^{k}$ admits a choking horn, namely the real part $X_{k}^{n} \cap(\mathbb{R} \times 0)^{n+1}$. 
Proof. Let

$$
X_{k}^{n}(\mathbb{R}):=\left\{\left(x_{1}, \ldots, x_{n}, y\right) \in \mathbb{R}^{n+1}: x_{1}^{2}+\ldots+x_{n}^{2}-y^{k}=0 \text { and } y \geq 0\right\}
$$

be the real algebraic variety embedded as the real part $X_{k}^{n} \cap \mathbb{R}^{n+1}$ of the hypersurface $X_{k}^{n}$, where $\mathbb{R}^{n+1} \subset \mathbb{C}^{n+1}$ is the usual embedding of $\mathbb{R}^{n+1}$ in $\mathbb{C}^{n+1}$. Consider the parameterization

$$
\phi:[0,1] \times \mathbf{S}^{n-1} \rightarrow X_{k}^{n}
$$

of the horn $X_{k}^{n}(\mathbb{R}) \subset \mathbb{C}^{n+1}$ defined by $\phi\left(t, u_{1}, \ldots, u_{n}\right)=\left(\left(t^{\frac{k}{2}} u_{1}, 0\right) \ldots,\left(t^{\frac{k}{2}} u_{n}, 0\right),(t, 0)\right)$ where $\mathbf{S}^{n-1}$ is the Euclidean unit sphere centered at origin of $\mathbb{R}^{n}$.

Claim 1. The $t$-link of $X_{k}^{n}(\mathbb{R})$ is not contractible in $X_{k}^{n} \backslash\{w=0\}$.

We start the proof of this claim by showing that for $0<t \leq 1$ the subset

$$
X_{k}^{n}(t):=\left\{\left(z_{1}, \ldots, z_{n}\right) \in \mathbb{C}^{n}: z_{1}^{2}+\ldots+z_{n}^{2}=t\right\}
$$

retracts within $\mathbb{C}^{n} \backslash\left\{z_{1}^{2}+\ldots+z_{n}^{2}=0\right\}$ to

$$
\left\{\left(z_{1}, \ldots, z_{n}\right) \in \mathbb{C}^{n}: z_{1}, \ldots, z_{n} \in \mathbb{R} \text { and } z_{1}^{2}+\ldots z_{n}^{2} \geq t\right\} .
$$

In fact, if we define $z_{j}:=u_{j}+i v_{j}$ with $u_{j}, v_{j} \in \mathbb{R}$, then

$$
z_{1}^{2}+\ldots+z_{n}^{2}=t \Leftrightarrow u_{1}^{2}+\ldots+u_{n}^{2}-\left(v_{1}^{2}+\ldots+v_{n}^{2}\right)=t \text { and } u_{1} v_{1}+\ldots+u_{n} v_{n}=0 .
$$

At the points $\left(z_{1}, \ldots, z_{n}\right)$ such that $z_{1}^{2}+\ldots+z_{n}^{2}=t$ we consider the retraction

$$
\left(s,\left(z_{1}, \ldots, z_{n}\right)\right) \longmapsto\left(u_{1}+i s v_{1}, \ldots, u_{n}+i s v_{n}\right) \text { where } 0 \leq s \leq 1 .
$$

Then

$$
\begin{aligned}
\left(u_{1}+i s v_{1}\right)^{2}+\ldots+\left(u_{n}+i s v_{n}\right)^{2} & =u_{1}^{2}+\ldots+u_{n}^{2}-s^{2}\left(v_{1}^{2}+\ldots+v_{n}^{2}\right)-2 i\left(u_{1} v_{1}+\ldots+u_{n} v_{n}\right) \\
& =t+\left(1-s^{2}\right)\left(v_{1}^{2}+\ldots+v_{n}^{2}\right) \\
& \geq t
\end{aligned}
$$

The proof of the claim will be finished once we have showed that the following subset

$$
\left\{\left(z_{1}, \ldots, z_{n}\right) \in \mathbb{C}^{n}: z_{1}, \ldots, z_{n} \in \mathbb{R} \text { and } z_{1}^{2}+\ldots+z_{n}^{2} \geq t\right\}
$$

retracts within itself to the subset

$$
\left\{\left(z_{1}, \ldots, z_{n}\right) \in \mathbb{C}^{n}: z_{1}, \ldots, z_{n} \in \mathbb{R} \text { and } z_{1}^{2}+\ldots+z_{n}^{2}=t\right\} .
$$

In fact, for each point $\left(z_{1}, \ldots, z_{n}\right) \in \mathbb{C}^{n}$ such that $z_{1}, \ldots, z_{n} \in \mathbb{R}$ and $z_{1}^{2}+\ldots+z_{n}^{2}=T \geq t$, we use the $\mathbb{R}_{+}$-action on $X_{k}^{n}$ to $\operatorname{map}\left(z_{1}, \ldots, z_{n}, T\right)$ to $\left(s^{k} z_{1}, \ldots, s^{k} z_{n}, s^{2} T\right)$ where $s=\sqrt[2 k]{\frac{t}{T}}$. Thus, we see that the subset

$$
X_{k}^{n}(t)=\left\{\left(z_{1}, \ldots, z_{n}\right) \in \mathbb{C}^{n}: z_{1}^{2}+\ldots+z_{n}^{2}=t\right\}
$$

retracts to $X_{k}^{n}(\mathbb{R})$ in $X_{k}^{n} \backslash\{w=0\}$. Let $g:\left(\mathbb{C}^{n}, \mathbf{0}\right) \rightarrow(\mathbb{C}, 0)$ be the function defined as $g\left(z_{1}, \ldots, z_{n}\right)=z_{1}^{2}+\ldots+z_{n}^{2}$. Since for non-zero $t$ the subset $X_{k}^{n}(t)$ is the Milnor Fiber of 
the function $g$, we deduce this subset is not contractible in $X_{k}^{n} \backslash\{w=0\}$. Thus Claim 1 is proved.

The next argument will complete the proof of the theorem. If $\xi_{t}$ is a chain with support contained in the $t$-link of $X_{k}^{n}$ and such that its boundary $\partial \xi_{t}$ is the $t$-link of $X_{k}^{n}(\mathbb{R})$, then

$$
\lim _{t \rightarrow 0} \frac{1}{t} \operatorname{diam}\left(\operatorname{supp}\left(\xi_{t}\right)\right) \geq \frac{\pi}{2}
$$

In particular, for $t>0$ small enough, we get $\operatorname{diam}\left(\operatorname{supp}\left(\xi_{t}\right)\right) \geq \frac{\pi}{4} t$.

Theorem 3.2. For each $n>1$ and any pair $k>l>1$, the complex isolated hypersurface singularities $X_{k}^{n}$ and $X_{l}^{n}$ are not subanalytically bi-Lipschitz homeomorphic.

Before getting into its proof, we note that Theorem 3.2 suggests that a local complex singularity topological type carry different local subanalytic inner Lipschitz geometries at the singular points.

Theorem 3.3. For any odd integer $n$, the family of germs $\left\{\left(X_{2 k+1}^{n}, \mathbf{0}\right)\right\}_{k>0}$ admits infinitely many subanalytically bi-Lipschitz classes, despite being of constant topological type.

Proof. When $n$ is odd Brieskorn [9] shows that the link at the origin of $X_{2 k+1}^{n}$ is a $\mathbb{Z}$ homology sphere, hence is homeomorphic to the Euclidean $(2 n-1)$-sphere. From Theorem 3.2, we deduce that the given family consists of infinitely many complex algebraic varieties which are (semialgebraically) homeomorphic but not subanalytically bi-Lipschitz homeomorphic.

Proof of Theorem 3.2. Suppose the contrary. So for some $k>l$, let $f: X_{k}^{n} \rightarrow X_{l}^{n}$ be a subanalytic bi-Lipschitz homeomorphism. Let us consider $X_{k}^{n}(\mathbb{R})$ the choking horn in $X_{k}^{n}$ and the parameterisation

$$
\phi:[0,1] \times \mathbf{S}^{n-1} \rightarrow X_{k}^{n}
$$

as defined in the proof of Theorem 3.1. So, the image of $f \circ \phi$, which we denote by $Y$, is a choking horn in $X_{l}^{n}$. We claim that the tangent cone of $Y$ at $\mathbf{0}$ is a real half-line in the $w$-complex axis (so we may take to be the $y$-real positive half-line after multiplying by $\left.e^{i \theta}\right)$. Before we prove what we claimed above, let us prove that if $\xi \in \mathbb{C}^{n+1}$ is a vector that does not lie in the $w$-axis, then for any sufficiently small conical neighborhood of $\xi$, which we denote by $C_{\epsilon}(\xi)$, there is at least one coordinate hyperplane $H_{i}=\left\{z_{i}=0\right\}$ such that the projection $X_{l}^{n} \cap C_{\epsilon}(\xi) \rightarrow H_{i}$ is subanalytically bi-Lipschitz. In fact, since $\xi \notin w$-axis at least one of the first coordinates of $\xi$ must differ from zero. Let us assume, without loss of generality, that $\xi_{1} \neq 0$ and let us consider the projection on $H_{1}=\left\{z_{1}=0\right\}$. In this case, locally on $X_{k}^{n}$ we have that $z_{1}$ is a function depending on $z_{2}, \ldots, z_{n}, w$ and

$$
\frac{\partial z_{1}}{\partial z_{i}}=-\frac{z_{i}}{z_{1}} \text { for } i=2, \ldots, n \text { and } \frac{\partial z_{1}}{\partial w}=\frac{k}{2} \frac{w^{k-1}}{z_{1}} .
$$


Since $z_{1}$ is bounded away from zero for all $z \in C_{\epsilon}(\xi)$, all partial derivatives above are bounded. We conclude that $z_{1}$ is a locally Lipschitz function, as we desired to show.

Since we have proved that some projection $X_{l}^{n} \cap C_{\epsilon}(\xi) \rightarrow H_{i}$ is subanalytically biLipschitz, if the tangent cone $T_{\mathbf{0}} Y$ were to be tangent to $\xi \notin w$-axis, we would have a choking horn in a metrically conical set which is impossible by Theorem 2.6.

Now, we know that $T_{\mathbf{0}} Y$ is the $y$-real positive half-line. Let us consider the following $\mathbb{R}_{+}$-action on $X_{l}^{n}$ :

$$
\left(t, z_{1}, \ldots, z_{n}, w\right) \longmapsto t \cdot\left(z_{1}, \ldots, z_{n}, w\right)=\left(t^{\frac{l}{2}} z_{1}, \ldots, t^{\frac{l}{2}} z_{n}, t w\right) .
$$

Let $u(t), v(t)$ be points belonging to $Y_{t}$. Then, we know that

$$
\|u(t)-v(t)\| \leq d_{X_{l}^{n}}(u(t), v(t)) \leq \lambda \operatorname{diam}\left(\left(X_{k}^{n}(\mathbb{R})\right)_{t}\right) \approx t^{\frac{k}{2}} \text { as } t \rightarrow 0 .
$$

Choosing the preimages of $u(t)$ and $v(t)$ so that their distance realizes the diameter of the $t$-link of $X_{k}^{n}$, we can use the $\mathbb{R}_{+}$-action on $X_{l}^{n}$ to push $Y_{t}$ to the 1 -slice of $X_{l}^{n}$. Then,

$$
u(t)=\left(u_{1}(t), \ldots, u_{n}(t), t\right) \text { and } v(t)=\left(v_{1}(t), \ldots, v_{n}(t), t\right)
$$

whence

$$
t^{-1} \cdot u(t)=\left(t^{-\frac{l}{2}} v_{1}(t), \ldots, t^{-\frac{l}{2}} u_{n}(t), 1\right) \text { and } t^{-1} \cdot v(t)=\left(t^{-\frac{l}{2}} v_{1}(t), \ldots, t^{-\frac{l}{2}} v_{n}(t), 1\right) .
$$

Hence

$$
\left\|t^{-1} \cdot u(t)-t^{-1} \cdot v(t)\right\| \leq \max _{i} t^{-\frac{l}{2}}\left|u_{i}(t)-v_{i}(t)\right| \lesssim t^{-\frac{l}{2}} t^{\frac{k}{2}} \rightarrow 0 \text { as } t \rightarrow 0
$$

since $k>l$.

But, then the 1-slice of $Y$ is homologous to a cycle that gets inside a contractible neighborhood in the 1-slice of $X_{l}^{n}$, so $Y$ cannot be a choking horn, which is a contradiction. This establishes the proof of the theorem.

\section{Further COMments}

Choking horns give an example of a structure that obstructs metric conicalness in any complex dimension greater than one. It seems likely that singular germs with a more sophisticated geometry should contain other objects (neither fast loops, nor separating sets nor choking horns) which are features of the local inner geometry at the singularity. A related series of questions comes from the fact that the choking horns we find above are the real part of a particular realization of the $X_{n}^{k}$ singularities. It is irresistible to ask whether one can detect failure of metric conicalness from the real parts of other realizations. On a related note, our proofs depended heavily on the $\mathbb{C}^{*}$ action on the singularity. Given a real singularity which is a horn, it is natural to ask whether its complexification must necessarily be metrically conic. A proof of anything along these lines would require very different techniques than ours. 


\section{Appendix: Separating Sets. By Walter D. Neumann}

This appendix describes a different proof of some of the results of this paper. We rely on the paper [6] of Birbrair, Fernandes and Neumann, in which is shown, among other things:

Theorem 5.1. If the tangent cone $T_{p} X$ of a normal complex germ $(X, p)$ has a complex subcone $V$ of complex codimension $\geq 1$ which separates $T_{p} X$, then there is a corresponding separating set in $(X, p)$ with tangent cone $V$.

The actual theorem in [6] is Theorem 5.1, which deals with the more general context of real semialgebraic sets, using a slightly more general definition of "separating set" than is needed in the complex setting. Here we define a separating set to be a real semialgebraic subgerm $(W, p) \subset(X, p)$ whose tangent cone has real codimension at least 2 and which separates $X$ into pieces whose tangent cones have full dimension.

The theorem is applied in [6] to the example of the Brieskorn variety

$$
X=X\left(a_{1}, \ldots, a_{n}\right):=\left\{\left(z_{1}, \ldots, z_{n}\right) \in \mathbb{C}^{n} \mid z_{1}^{a_{1}}+\cdots+z_{n}^{a_{n}}=0\right\}
$$

with $a_{1}=a_{2}=a \geq 2$ and $a_{k}>a$ for $k>2$. The tangent cone at the origin is the union of the a complex hyperplanes $\left\{z_{1}=\xi z_{2}\right\}$ with $\xi$ an $a$-th root of -1 . These intersect along the $(n-2)$-plane $V=\left\{z_{1}=z_{2}=0\right\}$, which separates the tangent cone into $a$ pieces. So $X$ has a separating set decomposing it into $a$ pieces having tangent cones the $a$ hyperplanes above. By Brieskorn [9], if $n>3$ then the link

$$
\Sigma\left(a_{1}, a_{2}, \ldots, a_{n}\right):=X\left(a_{1}, a_{2}, \ldots, a_{n}\right) \cap S^{2 n-1}
$$

is a topological sphere if at least two of the $a_{j}$ 's have no common factor with any other $a_{j}$. We thus see examples in any dimension $n \geq 3$ of singularities with link a topological sphere and having separating sets which decompose $X$ into arbitrarily many pieces. This gives a proof of Theorem 3.3 for all dimensions $\geq 3$.

In fact we can be very explicit in this example. Choose any positive $\epsilon<1$. Then putting

$$
Y:=\left\{\left.z \in X\left|\sum_{i=3}^{n}\right| z_{i}\right|^{a_{i}} \leq \epsilon\left(\left|z_{1}\right|^{a}+\left|z_{2}\right|^{a}\right)\right\}, \quad Z:=\overline{X \backslash Y}
$$

the following facts are easily verified:

(1) $T_{0} Z=\left\{z \in \mathbb{C}^{n} \mid z_{1}=z_{2}=0\right\}$;

(2) the image $\pi(Y)$ of the projection of $Y$ to the $z_{1} z_{2}$-plane has a components;

(3) the inverse image of each component of $\pi(Y)$ is a component of $Y$, so $Y$ has $a$ components. 
Indeed, (1) follows immediately from the fact that the exponents $a_{i}$ for $i \geq 3$ are greater than $a$. For $(2)$ we note that for any $z=\left(z_{1}, \ldots, z_{n}\right) \in Y$ :

$$
\begin{aligned}
\left|z_{1}^{a}+z_{2}^{a}\right| & =\left|\sum_{i=3}^{n} z_{i}^{a_{i}}\right| \leq \sum_{i=3}^{n}\left|z_{i}\right|^{a_{i}} \\
& <\left|z_{1}\right|^{a}+\left|z_{2}\right|^{a} .
\end{aligned}
$$

This inequality implies that the coordinates $z_{1}$ and $z_{2}$ are both non-zero, and $z_{2} / z_{1}$ cannot be a positive multiple of any $a$-th root of unity. This condition divides $\pi(Y)$ into $a$ pieces according to the argument of $z_{2} / z_{1}$. For each $a$-th root of -1 we denote by $Y_{\xi}^{\prime}$ the piece of $\pi(Y)$ which contains points with $z_{2} / z_{1}=\xi$ and denote $Y_{\xi}=\pi^{-1}\left(Y_{\xi}^{\prime}\right)$. It is not hard to check that $Y_{\xi}$ is connected and its projection to the hyperplane $z_{1}=0$ is a bijective map to its image which is subanalytically bi-Lipschitz in a neighbourhood of the origin. In fact, $(X, p)=(Y, p) \cup(Z, p)$, glued along their common boundary, which is topologically the cone over a disjoint union of $a$ copies of $S^{2 n-5} \times S^{1}$.

The previous decomposition $(X, p)=(Y, p) \cup(Z, p)$ is the "thick-thin decomposition" of $(X, p)$. This thick-thin decomposition is discussed in detail for normal surface singularities in [8] (works in progress about such a decomposition in any dimension by Birbrair, Fernandes, Grandjean, Neumann, O'Shea, Pichon, Verjovsky). In the case of an isolated complex singularity germ $(X, p)$ one can construct it as follows: call a tangent line $L$ in $T_{p} X$ "very exceptional" if no curve in $X$ with tangent $L$ has a metrically conical neighbourhood. One obtains the thin zone by taking a suitable horn neighbourhood of the union of all very exceptional tangent lines, and the thick zone is then the closure of the complement of the thin zone.

Similarly, the example $X(2, \ldots, 2, k) \subset \mathbb{C}^{n+1}$ of the body of this paper has thick-thin decomposition whose thin part is a $k / 2$-horn neighbourhood of the $z_{n+1}$-axis, which has boundary the cone over $S^{1} \times \Sigma(2, \ldots, 2)$.

\section{REFERENCES}

[1] L. Birbrair \& J.-P. Brasselet, Metric homology. Comm. Pure Appl. Math. 53 (2000), no. 11, 1434-1447.

[2] L. Birbrair \& J.-P. Brasselet, Metric homology for isolated conical singularities. Bull. Sci. Math. 126 (2002), no. 2, 87-95.

[3] L. Birbrair \& A. Fernandes, Inner metric geometry of complex algebraic surfaces with isolated singularities. Comm. Pure Appl. Math. 61 (2008), no. 11, 1483-1494.

[4] L. Birbrair \& A. Fernandes \& W. Neumann, Bi-Lipschitz geometry of weighted homogeneous surface singularities. Math. Ann. 342 (2008), no. 1, 139-144.

[5] L. Birbrair \& A. Fernandes \& W. Neumann, Bi-Lipschitz geometry of complex surface singularities. Geom. Dedicata 139 (2009), 259-267.

[6] L. Birbrair \& A. Fernandes \& W. Neumann, Separating sets, metric tangent cone and applications for complex algebraic germs. Selecta Math. (N.S.) 16 (2010), no. 3, 377-391. 
[7] L. Birbrair \& T. Mostowski, Normal embeddings of semialgebraic sets. Michigan Math. J. 47 (2000), no. 1, 125-132.

[8] L. Birbrair \& W. Neumann \& A. Pichon, The thick-thin decomposition and the bi-Lipschitz classification of normal surface singularities. arXiv:1105.3327v3.

[9] E. Brieskorn, Examples of singular normal complex spaces which are topological manifolds. Proc. Nat. Acad. Sci. U.S.A. 55 (1966), 1395-1397.

[10] A. Fernandes, Fast loops on semi-weighted homogeneous hypersurface singularities. J. Singul. 1 (2010), 85-93.

[11] K. Kurdyka, On a subanalytic stratification satisfying a Whitney property with exponent 1. Real algebraic geometry (Rennes, 1991), 316322, Lecture Notes in Math., 1524, Springer, Berlin, 1992.

[12] K. Kurdyka, P. Orro Distance géodésique sur un sous-analytique. Real algebraic and analytic geometry (Segovia, 1995). Rev. Mat. Univ. Complut. Madrid 10 (1997), Special Issue, suppl., 173182.

[13] T. Mostowski, Lipschitz equisingularity. Dissertationes Math. (Rozprawy Mat.) 243 (1985), 46 pp.

[14] A. Parusiński, Lipschitz stratification of subanalytic sets. Ann. Sci. École Norm. Sup. (4) 27 (1994), no. 6, 661-696.

[15] G. Valette, Vanishing Homology. Selecta Math. (N.S.) 16 (2010), no. 2, 267-296.

Departamento de Matemática, Universidade Federal do Ceará (UfC), Campus do PiCici, Bloco 914, CeP. 60455-760. Fortaleza-Ce, Brasil

E-mail address: birb@ufc.br

Departamento de Matemática, Universidade Federal do Ceará (UfC), Campus do PiCici, Bloco 914, Cep. 60455-760. Fortaleza-Ce, Brasil

E-mail address: alexandre.fernandes@ufc.br

Departamento de Matemática, Universidade Federal do Ceará (UfC), Campus do PiCici, Bloco 914, CeP. 60455-760. Fortaleza-Ce, Brasil

E-mail address: vgrandje@fields.utoronto.ca

President's Office, New College of Florida, 5800 Bayshore Road, Sarasota, FL 34243, USA

E-mail address: doshea@ncf .edu 